\title{
Caso 6/2008 - Lactente de 13 Meses, do Sexo Masculino, com Vaso Colateral Sistêmico-Pulmonar para o Lobo Inferior Esquerdo, com Possível Fístula Arteriovenosa nesse mesmo Lobo, com Coração Estruturalmente Normal
}

\author{
Arteriovenous Fistula in the same Lobe, with a Structurally Normal Heart \\ Edmar Atik \\ Instituto do Coração da Faculdade de Medicina da Universidade de São Paulo - São Paulo, SP - Brasil
}

Case 6/2008 - Thirteen-month-old Male Infant, with Systemic-Pulmonary Collateral Vessel into the Left Inferior Lobe, with Possible

\section{Dados clínicos}

Descoberto cardiomegalia em radiografia de tórax em vigência de pneumonia, com sete meses de idade. Cansaço discreto foi notado desde então, mantendo-se sem medicação.

\section{Exame físico}

Taquipnéia discreta, acianótico (saturação capilar de 98\%) e pulsos normalmente palpados nos quatro membros. Peso: $12 \mathrm{~kg}$, altura: $78 \mathrm{~cm}$. PA: 90/60 mmHg. FC: 128 bpm. Aorta não era palpada na fúrcula.

No precórdio não havia deformidades ou impulsões. O ictus cordis foi palpado no 4o espaço intercostal esquerdo na linha hemiclavicular e limitado por uma polpa digital. A 2a bulha cardíaca era discretamente hiperfonética na área pulmonar e foi auscultado sopro sistólico, de ejeção, na área mitral e no dorso à esquerda.

Os pulmões estavam bem aerados e sem ruídos adventícios, e o fígado era palpado a $1,5 \mathrm{~cm}$ do rebordo costal direito.

O eletrocardiograma mostrava ritmo sinusal e sinais de sobrecarga da cavidade ventricular esquerda, dada a presença de ondas QR acentuadas de V4 a V6, com ÂQRS a $+40^{\circ}$, $\hat{\mathrm{AP}}:+60^{\circ}$ e $\hat{\mathrm{AT}} \mathrm{T}:+10^{\circ}$.

\section{Imagem radiográfica}

Mostra área cardíaca de dimensão discretamente aumentada, com arco ventricular esquerdo alongado e arredondado e com trama vascular pulmonar normal. O arco médio está escavado. Chama a atenção uma imagem

\section{Palavras-chave}

Fístula arteriovenosa, cardiomegalia, insuficiência da valva mitral.

\section{Correspondência: Edmar Atik •}

InCor - Av. Dr. Enéas Carvalho de Aguiar, 44 - 05403-000 - São Paulo, SP - Brasil

E-mail: conatik@incor.usp.br hipertransparente, no lobo inferior esquerdo e retrocardíaca, que salienta nesse local a trama vascular pulmonar mais proeminente e de bordos pouco nítidos (fig.1).

\section{Impressão diagnóstica}

Essa imagem radiográfica é compatível com o diagnóstico de possíveis fístulas pronunciadas arteriovenosas pulmonares no lobo inferior esquerdo, ou ainda por vasos sistêmico-pulmonares, com conseqüente aumento ventricular esquerdo.

\section{Diagnóstico diferencial}

Aumento de ventrículo esquerdo com trama vascular pulmonar normal poderia também orientar para cardiopatia com insuficiência valvar mitral ou mesmo para miocardiopatia dilatada.

\section{Confirmação diagnóstica}

Os elementos clínicos foram compatíveis com o diagnóstico inicial de insuficiência mitral, corroborados com o ecocardiograma que salientava aumento discreto das cavidades esquerdas. No entanto, não havia sinais de regurgitação no nível da valva mitral, mas fluxo sistêmicopulmonar em aorta descendente por possível vaso colateral. Angiotomografia mostrou vaso colateral calibroso com 7 $\mathrm{mm}$ de diâmetro na porção inferior da aorta descendente, que continuava com a artéria lobar inferior esquerda. A partir daí, visibilização de vasos calibrosos e tortuosos no lobo inferior esquerdo e com drenagem rápida pela veia pulmonar inferior ao átrio esquerdo (fig.2). O cateterismo cardíaco mostrou esse mesmo aspecto angiográfico, com fluxo de retorno venoso pulmonar rápido para as cavidades esquerdas (fig.2). A cintilografia pulmonar com tecnésio em injeção venosa mostrou a falta de mapeamento no lobo inferior esquerdo, sendo o fluxo pulmonar direito de $60 \%$, o pulmonar esquerdo de $30,2 \%$, e o fluxo sistêmico de $9,7 \%$. Injeção seletiva do radioisótopo diretamente na colateral sistêmico-pulmonar revelou que 30\% desse fluxo se desviava para o lado sistêmico caracterizando possivelmente a presença de fístula arteriovenosa nesse lobo. 


\section{Correlação Clínico-Radiográfica}

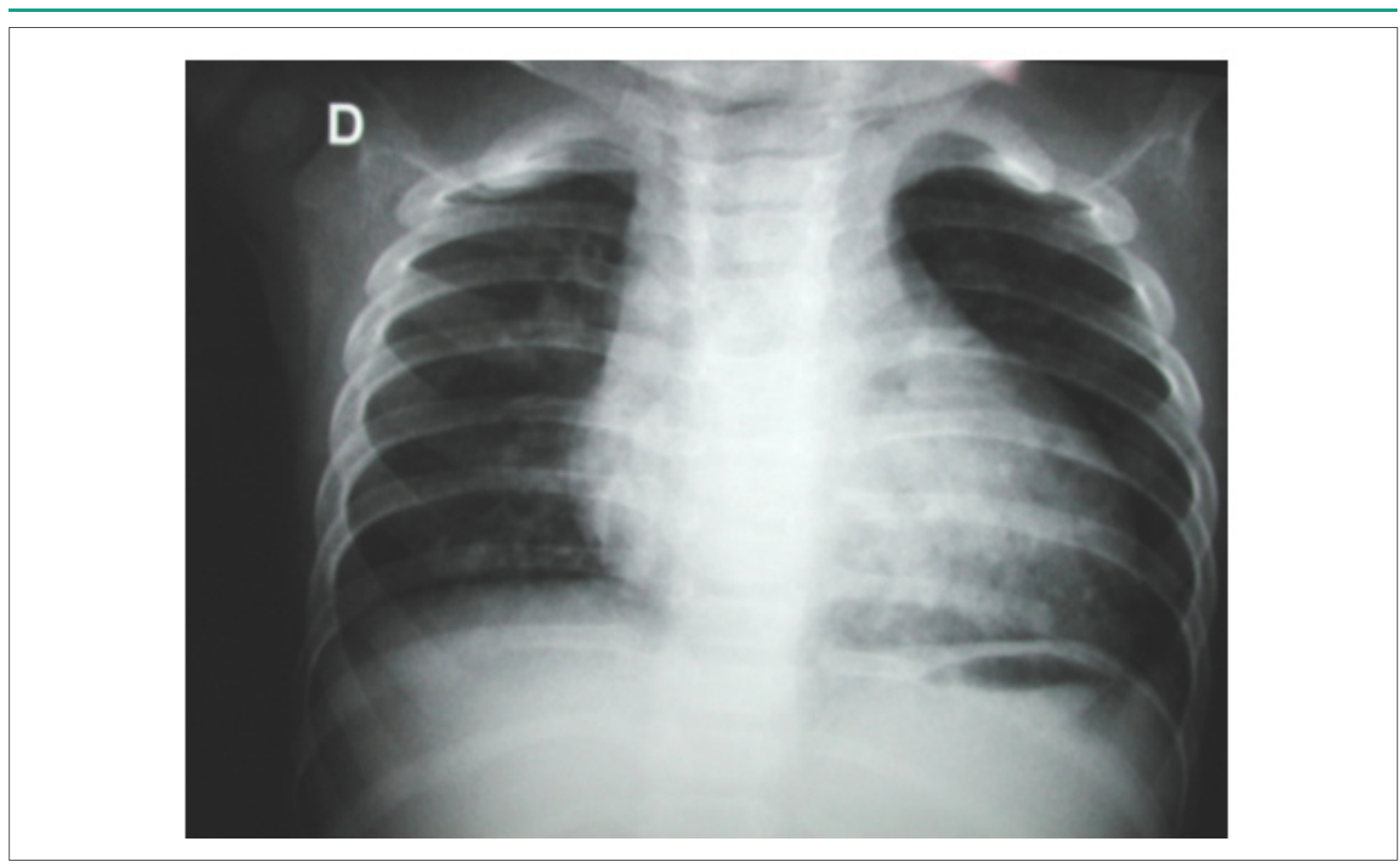

Fig. 1 - Radiografia de tórax mostra área cardíaca discretamente aumentada, com arco ventricular esquerdo alongado e arredondado; A trama vascular pulmonar é normal; É nítida a imagem de condensação retrocardíaca à esquerda, no lobo inferior, compatível com fístula arteriovenosa.
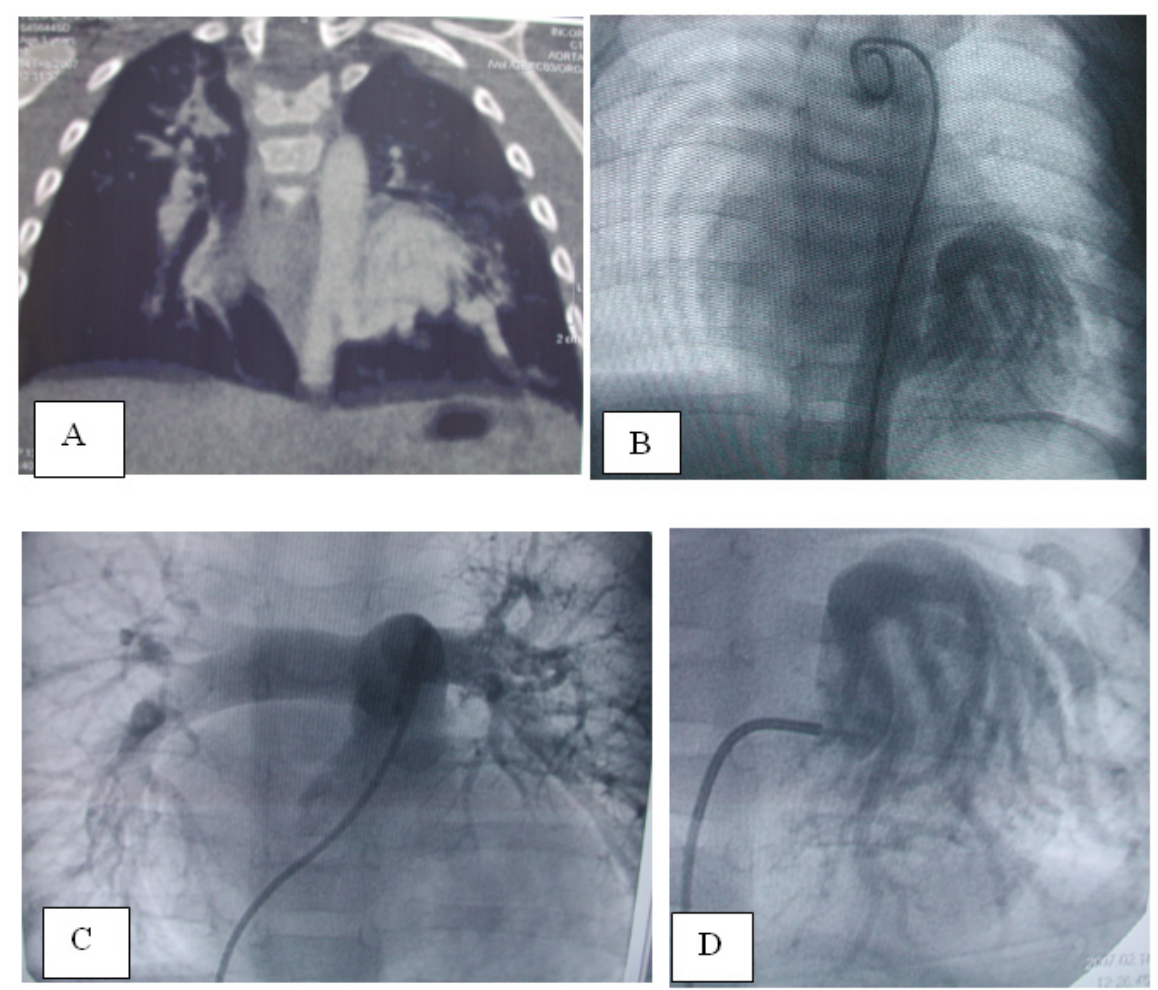

Fig. 2 - Imagens com contraste salientam a presença de vaso colateral sistêmico-pulmonar nascendo da aorta descendente em A (angiotomografia) e em B (angiografia arterial), com vasos arteriovenosos tortuosos e calibrosos, exteriorizados principalmente em $D ; A$ injeção na artéria pulmonar salienta a ausência do enchimento do lobo inferior esquerdo, em C. 


\section{Correlação Clínico-Radiográfica}

\section{Conduta}

Dada a desconexão com a árvore arterial pulmonar, ademais da fístula arteriovenosa pulmonar, foi realizada a lobectomia pulmonar inferior esquerda, após a dissecção e ligadura do vaso sistêmico pulmonar, da artéria lobar inferior esquerda e do brônquio inferior esquerdo. Biópsia pulmonar desse lobo revelou hipertensão arterial pulmonar, grau IV de Heath-Edwards. A evolução foi boa.

\section{Considerações}

Sem cardiopatia demonstrada, mas com fístulas arteriovenosas pulmonares, a ausência de cianose pode ser explicada por desvio arteriovenoso de sangue já saturado desde a aorta para o lobo inferior esquerdo, o que orienta em última análise para um tipo de seqüestro pulmonar. Dada a magnitude desse desvio de sangue, explica-se daí o aumento do ventrículo esquerdo na radiografia de tórax e a sobrecarga ventricular esquerda no eletrocardiograma. A lobectomia pulmonar foi considerada mais eficaz e de menor risco que a conexão da artéria lobar inferior esquerda com o restante da árvore arterial pulmonar, ainda mais que havia acentuado e irreversível grau de hipertensão pulmonar. 